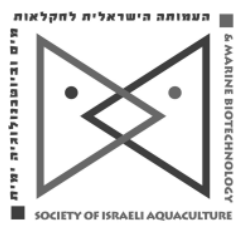

The IJA appears exclusively as a peerreviewed on-line Open Access journal at http://www.siamb.org.il Sale of IJA papers is strictly forbidden.

\title{
Efficacy of an Inactivated Vaccine and Nutritional Additives Against White Spot Syndrome Virus (WSSV) in Shrimp (Penaeus monodon)
}

\author{
Edgar C. Amar* and Joseph P. Faisan Jr. \\ Aquaculture Department, Southeast Asian Fisheries Development Center, \\ Tigbauan, Iloilo, 5021, Philippines \\ (Received 4.2.10, Accepted 8.3.10)
}

Key words: Penaeus monodon, WSSV, formalin-killed vaccine, immunity, nutrition, antioxidants, wheat grass, methyl sulfonyl methane, alfalfa

\begin{abstract}
Although the immune system of shrimps is not comparable to that of vertebrates, shrimps can acquire protection against pathogenic challenge by building up immunity. In this study, formalininactivated virus (FIV) was administered by injection, bathimmersion, or orally to determine levels of vaccination-mediated protection against the pathogenic white spot syndrome virus (WSSV). Diets supplemented with alfalfa, methyl sulfonyl methane (MSM), or wheat grass were provided with or without FIV. Shrimp injected with FIV and challenged 3, 15, or 30 days after vaccination had cumulative and relative survivals of $83 \%, 67 \%$, and $33 \%$, respectively. Survival of shrimp challenged by bathimmersion 3-45 days after vaccination by immersion was significantly higher than in the unvaccinated control. Orally vaccinated shrimp challenged by bath-immersion were partially protected up to 45 days after vaccination (cumulative survival $63.7 \%$, relative $61.7 \%$ ) but not til 60 days after vaccination (cumulative $8 \%$, relative $3.2 \%$ ). Survival of unvaccinated shrimp challenged by bath-immersion improved when shrimp were fed a diet supplemented with wheat grass or MSM, but not alfalfa. Survival was further enhanced when FIV was provided together with diets supplemented with wheat grass (cumulative $72.7 \%$, relative $94.8 \%$ ) or MSM (cumulative $73.3 \%$, relative $96.3 \%$ ).
\end{abstract}

* Corresponding author. E-mail: eamar@seafdec.org.ph 


\section{Introduction}

Viruses are among the greatest threats to the worldwide shrimp aquaculture industry. To be essentially virus-free, shrimp must be reared during all life stages in a bio-secure environment that prevents contact with possible vectors in the natural environment (Flegel, 2009). However, this would entail domestication and selection programs that require long gestation periods and huge capital investments. Immunostimulation and vaccination are widely used in mammals and other vertebrates. But vaccination, considered the gold standard in disease prevention for vertebrates, is considered unsuitable for invertebrates such as shrimps which are thought to possess only innate immunity and not have the ability to produce antibodies.

Nevertheless, Penaeus monodon and $P$. japonicus have been vaccinated against vibriosis (Teunissen et al., 1998). The plasma of shrimp exposed to inactivated white spot syndrome virus (WSSV) or its sub-units contains virusneutralizing activity (Venegas et al., 2000) and exhibits reduced mortality upon challenge (Namikoshi et al., 2004; Bright Singh et al., 2005), suggesting the presence of an inducible immunity that can inhibit subsequent infection by the same pathogen. Previous exposure to a recombinant viral envelop protein (rVP28) but not to an unrelated protein can also protect shrimp from WSSV (Witteveldt et al., 2004; Caipang et al., 2008). Experiments with other crustaceans such as Daphnia show that it is capable of memory and specificity (Kurtz, 2004) and a highly varied recognition capacity has been found in insects (Watson et al., 2005).

In all these studies, however, no evidence was presented to explain the protective mechanism. The underlying mechanisms in shrimp vaccination are just beginning to be addressed (Johnson et al., 2007). The discovery of induced protective factors can be facilitated by expression studies of immunity-related genes (Leu et al., 2007).

Study of the shrimp immune response is necessary to develop methods for increasing survival and overall disease resistance and reducing the impact of WSSV infection. In this study, we compare the efficacy of various routes of vaccination using inactivated whole viral particles and of dietary additives with antioxidant properties in enhancing the efficacy of formalin-inactivated vaccines in shrimp. The shrimp were challenged by WSSV in several ways and the effects of administering naturally-occurring or plant-derived immunomodulatory substances with the inactivated vaccine on shrimp resistance to WSSV were examined.

\section{Materials and Methods}

Experimental animals. Since specific pathogen-free (SPF) Penaeus monodon were not available, shrimp juveniles (10-15 g) were obtained from a farm that routinely implements biosecurity measures and had no history of WSSV outbreak. Samples were analyzed by bacterial plating of tissue homogenates and polymerase chain reaction (PCR) to confirm the absence of pathogens such as luminescent Vibrio and WSSV. Shrimp were stocked at 15 animals/100-I fiberglass tank supplied with UV-filtered sea water and adequate aeration. The shrimp were acclimated to tank conditions in 
SEAFDEC/AQD's experimental infection facilities (also biosecure) for three weeks before the start of the experiment. During acclimation they were fed a SEAFDEC/AQD formulated shrimp grow-out diet (FDS, 1994). Ambient water temperature ranged $28-30^{\circ} \mathrm{C}$ and salinity fluctuated $28-32$ ppt. The shrimp were determined to be fully acclimated when they resumed normal feeding and had no body lesions. PCR-screened shrimp postlarvae (PL) were obtained from a private hatchery with biosecure facilities, quarantined for two weeks and grown to 3-5 $\mathrm{g}$ before being used in the bath-immersion and oral vaccination trials.

Preparation of formalin-killed vaccine. Purified virus was prepared from frozen and pooled gill tissues ( $3 \mathrm{~g}$ for intramuscular injection and $5 \mathrm{~g}$ for oral vaccination) excised from moribund experimentally-infected shrimp. The tissues were homogenized in TN Buffer and centrifuged at $3000 \mathrm{~g}$. The supernatant fluid was collected with a pipette and layered carefully on top of a $30 \%$ sucrose gradient. The tubes with the fluid were centrifuged at 30,000 RPM in an Optima ${ }^{\mathrm{TM}} \mathrm{L}-100 \mathrm{~K}$ preparative ultracentrifuge (Beckman Coulter, Inc., Fullerton, CA) for $3 \mathrm{~h}$ at $4^{\circ} \mathrm{C}$. The virus bands were collected with a pipette, washed once, and pelleted at 40,000 RPM for $2 \mathrm{~h}$. The virus pellets were then resuspended in TN Buffer and inactivated by overnight incubation in $0.5 \%$ formalin. After removal of the formalin and resuspension in phosphate buffered saline (PBS), the inactivated virus was diluted 10 times and used directly for injection, diluted in water for bath-immersion, or added to the formulated diet for oral delivery.

Vaccine delivery. For intramuscular injection (IM), shrimp were removed from the experimental tanks and transferred to aerated basins. Each shrimp was wrapped in a paper towel and placed on top of an ice gel for a few seconds to immobilize it. The formalin-inactivated vaccine ( $30 \mu \mathrm{g} / \mathrm{shrimp})$ was carefully injected into each shrimp on the ventral right side of the second abdominal segment using a 1-ml tuberculin syringe fitted with a gauge 30 needle (Terumo Philippines Corp., Laguna, PH). Injected shrimp were immediately returned to their respective tanks. For immersion, $10 \mathrm{ml}$ of the vaccine was dispensed with a micropipette and diluted to $10^{-4}$ in $5-\mathrm{I}$ aerated seawater aquaria. After the vaccine was evenly dispersed in the water, shrimp were removed from the culture tanks and immersed in the aquaria. After $2 \mathrm{~h}$ of exposure, the shrimp were returned to the culture tanks. For oral delivery, the SEAFDEC/AQD formulated diet was modified by adding methyl sulfonyl methane (MSM; Vitabasix LHP, Inc.), wheat grass (Platinum HP, CA), or alfalfa powder (Nature Inc.) at $1 \mathrm{~g} / 100 \mathrm{~g}$ of the dry diet to serve as immunomodulators. The liquid vaccine was hand-sprayed on the feed pellets at a final concentration of $5 \mathrm{ml}$ vaccine/500 $\mathrm{g}$ dry diet ( $50 \mu \mathrm{g}$ vaccine/shrimp) and allowed to absorb into the pellets. The pellets were air dried and coated with a film of cod liver oil to prevent leaching of the vaccine into the water. The feed was dried with $\mathrm{N}_{2}$ gas in sealable plastic bags and stored at $4^{\circ} \mathrm{C}$ between each feedings.

Five replicate groups of 15 shrimp, each, received the IM injection or the oral treatment; six groups of 15 shrimp, each, were vaccinated by bath- 
immersion. In the supplemented feed experiment, 15 shrimp $(4 \mathrm{~g})$ in each of three replicates for each of nine treatments (405 animals in 27 tanks) were fed a treated or control diet for 14 days before being challenged with live virus by bath-immersion. Precautions were taken to prevent unnecessary handling stress to the shrimp. Thus, shrimp in the corresponding shamvaccinated controls were handled similarly as shrimp vaccinated by IM injection and bath immersion.

Challenge trials. A challenge experiment was performed following every vaccination trial. For IM injection challenge, the virus was isolated from $1 \mathrm{~g}$ of pooled gill tissues from experimentally infected shrimps that showed definite signs of viral infection and were PCR-positive. The gill tissues were homogenized and centrifuged, and the supernatants were collected, filtered through a 0.45- $\mu \mathrm{m}$ cellulose membrane filter (Advantec Toyo Kaisha Ltd., Tokyo, Japan), and used as an inoculum. The challenge dose $\left(L_{50}\right)$, i.e., the concentration required to kill $50 \%$ of the shrimp, was determined in a preliminary in vivo titration experiment using tissue filtrate dilutions ranging $10^{-3}$ to $10^{-9}$ and was determined to be $10^{-6.45}$. A stock dilution of $10^{-2}$ was prepared in PBS and frozen for subsequent challenges. Shrimp were challenged by IM injection 3, 15, and 30 days after vaccination and survival in each group was monitored for 15 days. For the bath-immersion challenge, the inoculum was prepared as above but diluted to $10^{-4}$ in 5 liters of water. Shrimp were challenged 3, 15, 30, and 45 days after vaccination by bathimmersion and 30,45 , and 60 days after oral vaccination. Survival was observed for 15 days. Shrimp in the mock-challenged controls were handled in the same way as those challenged with the viral inoculum.

Statistical analysis. Data were analyzed with one-way ANOVA followed by Tukey's HSD test (SYSTAT 8.0, SPSS, Chicago, IL). Mean differences were considered significant when $p<0.05$. Final cumulative mortality values were arc sine transformed before being analyzed by ANOVA and Tukey's test. Relative survival was calculated according to Amend (1981) when differences between the control and treatment groups were significant.

\section{Results}

Shrimp challenged by IM injection 3 and 15 days after IM vaccination had the highest cumulative survival, $83 \%$ and $67 \%$, respectively (Fig. 1). Shrimp challenged 30 days after IM vaccination had $33 \%$ cumulative survival. Shrimp challenged by bath-immersion after vaccination by bath-immersion had significantly higher survival than the mock-vaccinated control (Fig. 2); survival was highest when shrimp were challenged three days after vaccination (cumulative $86 \%$, relative $77.6 \%$ ) and lowest when challenged 45 days after vaccination (cumulative $66 \%$, relative $46.3 \%$ ). Shrimp challenged by bath-immersion were partially protected up to 45 days after oral vaccination (cumulative $63.7 \%$, relative $61.7 \%$ ), but survival did not significantly differ from the control when challenged 60 days (cumulative $8 \%$, relative $3.2 \%$ ) after vaccination (Fig. 3 ).

Shrimp given diets supplemented with wheat grass or methyl sulfonyl methane (MSM) survived WSSV challenge better than those fed the control 
diet (Fig. 4). However, relative survival was only $28.7 \%, 18.4 \%$, and $27.9 \%$ for wheat grass, alfalfa, and MSM, respectively. Vaccinated shrimp had better survival (cumulative $60.7 \%$, relative $68.4 \%$ ) than unvaccinated and survival was best in the FIV+ wheat grass (cumulative $72.7 \%$, relative $94.8 \%$ ) and FIV+MSM groups (cumulative $73.3 \%$; relative $96.3 \%$ ).

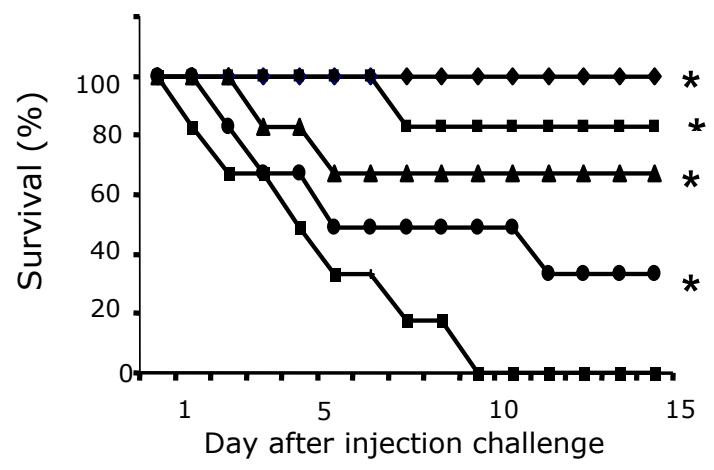

Fig. 1. Cumulative survival in Penaeus monodon juveniles vaccinated by intramuscular injection and challenged by injection of $50 \mu \mathrm{l}$ of a $10^{-6.45}$ dilution of WSSV inoculum. $=$ mockvaccinated and mock-challenged control; . = challenged three days after vaccination, $\boldsymbol{\Delta}=15$ days after vaccination, $\bullet=30$ days after vaccination; = mock-vaccinated challenged control. Asterisk indicates significant difference from mock-vaccinated challenged control $(p<0.05)$.

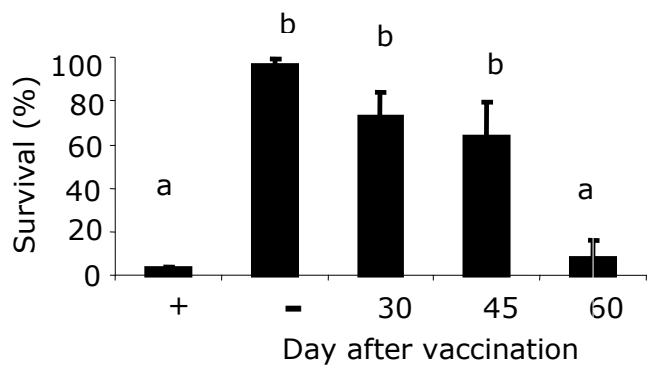

Fig. 3. Survival of Penaeus monodon juveniles challenged by bath-immersion 30, 45, or 60 days after oral vaccination. $+=$ mockvaccinated challenged control; - = mockvaccinated and mock-challenged control. Means with different letters significantly differ $(p<0.05)$.

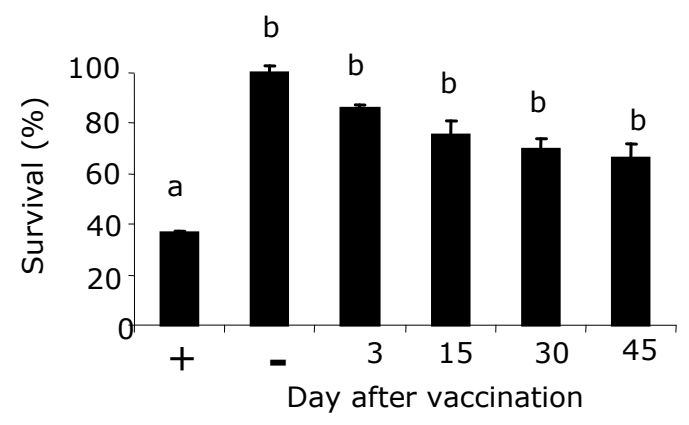

Fig. 2. Survival of Penaeus monodon juveniles vaccinated by bath-immersion and challenged by immersion in a $10^{-4}$ dilution of WSSV viral inoculum. $+=$ mock-vaccinated challenged control; - = mock-vaccinated and mock-challenged control. Means with different letters significantly differ $(p<0.05)$.

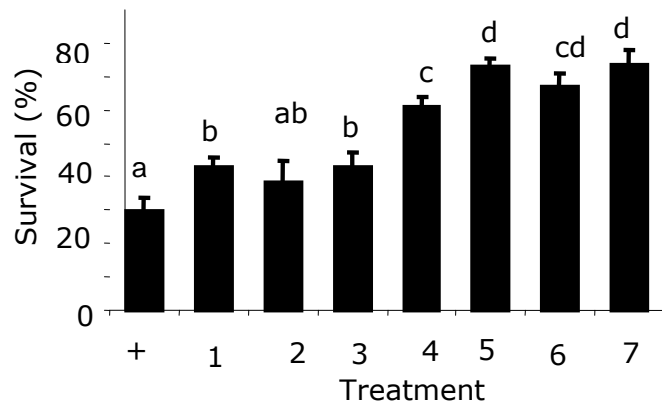

Fig. 4. Survival of Penaeus monodon juveniles fed diets with or without immunomodulatory substances, sprayed with formalin-killed vaccine (FIV). Shrimp were challenged by bath-immersion in a $10^{-4}$ dilution of WSSV inoculum. $+=$ unsupplemented control; $1=$ wheat grass; $2=$ alfalfa; $3=$ methyl sulfonyl methane (MSM); 4 = FIV; $5=$ FIV+wheat grass; $6=$ FIV+alfalfa; $7=$ FIV+MSM. Negative control had no mortality and is not shown. 


\section{Discussion}

Shrimp vaccinated by IM injection were partially protected from challenge by IM injection of the WSSV virus for up to 30 days. Shrimp that were vaccinated by bath-immersion or orally were significantly protected from challenge by bath-immersion for up to 45 days. Based on relative survival values, significant protection $(\geq 60 \%$ ) was obtained for only 15 days when shrimp were challenged by injection and up to 45 days when challenged by bathimmersion, regardless of the method of vaccination. The longer protection obtained when challenged by bath-immersion in both bath-immersed and orally-vaccinated shrimp indicates that it is a more benign challenge method than IM injection.

Our results are consistent with observations in $P$. monodon (Witteveldt et al., 2004), P. japonicus (Namikoshi et al., 2004), and Fenneropenaeus indicus (Bright Singh et al., 2005) obtained with formalin-killed WSSV and VP28 vaccines. These earlier results, although not directly comparable, led the above investigators to postulate the presence of 'adaptive' immunity in shrimp. Highly specific immune responses have been found in mollusks and arthropods in which immunoglobulin superfamily (IgSf) proteins such as Down's syndrome cell adhesion molecule (DSCAM) and fibrinogen-related protein (FREP) exist in variable isoforms produced by alternative splicing (Zhang and Loker, 2003; Brites et al., 2008).

Multiple mechanisms for generating highly specific innate immune responses in invertebrates have been proposed (Schulenberg et al., 2007). The mechanism of induced protection against viruses in penaeid shrimp, however, is not explicitly known. Based on empirical data and studies in insects, Flegel (2007) advocated the viral accommodation concept where the shrimp adapts and develops tolerance to the virus to accommodate persistent infections. Adaptive tolerance rather than resistance would favor the continued survival of the host species by removing pressure on the pathogen to evolve into strains that are more virulent. In an update of antiviral immunity in crustaceans, induced genes associated with the ability of shrimp to survive viral infections have been reported, but whether they lead to the production of antiviral substances has not been clearly demonstrated (Liu et al., 2009). Moreover, induced antiviral resistance in shrimp can also be elicited by sequence-specific RNA interference mechanisms (Robalino et al., 2007). In any event, the short duration of protection may suggest a need for booster vaccinations and suitably administered feed-based additives to enhance the efficacy of the vaccine.

MSM and wheat grass enhanced survival to some extent, but offered no real benefit in terms of relative survival to unvaccinated shrimp. Synergistic effects were evident when MSM or wheat grass and FIV were co-administered to the shrimp before bath-immersion challenge. During infection, sustained production of immune effectors by an activated immune system may result in depletion of energy stores and immune fatigue that can be harmful to the host (Moret and Scmid-Hempel, 2000). Viral infections are known to induce excessive production of reactive oxygen species (ROS), resulting in imbalance in the antioxidant and pro-oxidant status of the host, i.e., oxidative stress. 
WSSV infection has been reported to deplete the antioxidant scavenger system in F. indicus (Mohankumar and Ramasamy, 2006). This can induce cell damage in target tissues, including cells of the immune system, and impairment of immune responses.

While low doses of ROS can cause cell proliferation, medium and high doses can lead to temporary or permanent growth arrest and cell death by apoptosis or necrosis (Holbrook and Ikeyama, 2002). Widespread apoptosis correlated with mortality occurs after infection by yellow head virus (YHV) and to some extent by WSSV in P. monodon (Khanobdee et al., 2002). The detrimental effects of oxidative stress, however, can be counteracted, and immune responses enhanced, by nutrient fortification or food components with antioxidant properties such as vitamins $C$ and $E$, flavonoids, and carotenoids (Ortuño et al., 2000; Amar et al., 2004).

Sulfur-containing compounds are generally considered antioxidants. MSM supplementation induces an increase in endogenous antioxidant enzyme glutathione peroxidase (GPX) levels as MSM metabolism provides one of the precursors of GPx synthesis (Marañon et al., 2008), whereas the phenolic and flavonoid contents in wheat grass account for its antioxidant activity (Kulkarni et al., 2006). Thus, the enhancing effects of MSM and wheat grass on vaccine efficacy in the present study were presumably due to their anti-inflammatory and antioxidant properties. This indicates that the benefits of vaccination can be optimized by mitigating the effects of oxidative stress and apoptosismediated mortality in shrimp through supplementation with dietary antioxidants. It is concluded that nutritional additives such as wheat grass and methyl sulfonyl methane can be co-administered with formalin-killed vaccines to strengthen immunity against WSSV in shrimp.

\section{Acknowledgments}

This study was supported by research funds from SEAFDEC/AQD and the Regional Fish Disease Project of the Government of Japan Trust Fund Phase IV under Study Code 8001-T-FD-FH1204.

\section{References}

Amar E.C., Kiron V., Satoh S. and T. Watanabe, 2004. Enhancement of innate immunity associated with dietary intake of carotenoids from natural products. Fish Shellfish Immunol., 16:527-537.

Amend D.F., 1981. Potency testing of fish vaccines. Dev. Biol. Standard., 49:447-454.

Bright Singh I.S., Manjusha M., Somnath Pai S. and S. Philip, 2005. Fenneropenaeus indicus is protected from white spot disease by oral administration of inactivated white spot syndrome virus. Dis. Aquat. Org., 66:265-270.

Brites D., McTaggart S., Morris K., Anderson J., Thomas K., Colson I., Fabbro T., Little T., Ebert D. and L. Du Pasquier, 2008. The Dscam homologue of the crustacean Daphnia is diversified by alternative splicing like in insects. Mol. Bio. Evol., 25:1429-1439. 
Caipang C.M.A., Verjan N., Ooi E.L., Kondo H., Hirono I., Aoki T., Kiyono H. and Y. Yushikazu, 2008. Enhanced survival of shrimp, Penaeus (Marsupenaeus) japonicus from white spot syndrome disease after oral administration of recombinant VP28 expressed in Brevibacillus brevis. Fish Shellfish Immunol., 25:315-320.

FDS, 1994. Feeds and Feeding of Milkfish, Nile Tilapia, Asian Seabass, and Tiger Shrimp. Aquaculture Extension Manual No. 21. Feed Development Section, SEAFDEC Aquacult. Dept., Tigbauan, Iloilo, Philippines. 97 pp.

Flegel T.W., 2007. Update on viral accommodation, a model of host-viral interaction in shrimp and other arthropods. Dev. Comp. Immunol., 31:217231

Flegel T.W., 2009. Current status of viral diseases in Asian shrimp aquaculture. Isr. J. Aquacult. - Bamidgeh, 61(3):229-239.

Holbrook N.J. and S. Ikeyama, 2002. Age-related decline in cellular response to oxidative stress: links to growth factor signaling pathways with common defects. Biochem. Pharmacol., 64:999-1005.

Johnson K.N., van Hulten M.C.W. and A.C. Barnes, 2008. "Vaccination" of shrimps against viral pathogens: phenomenology and underlying mechanisms. Vaccine, 26:4885-4892.

Khanobdee K., Soowanayan C., Flegel T.W., Ubol S. and B. Withyachumnarnkul, 2002. Evidence for apoptosis correlated with mortality in the giant black tiger shrimp Penaeus monodon infected with yellow-head virus. Dis. Aquat. Org., 48:79-90.

Kulkarni S.D., Tilak J.C., Acharya R., Rajurkar N.S., Devasagayam T.P.A. and A.V.R. Reddy, 2006. Evaluation of the antioxidant activity of wheatgrass (Triticum aestivum L.) as a function of growth under different conditions. Phytotherapy Res., 20:218-227.

Kurtz J., 2004. Memory in the innate and adaptive immune systems. Microbes Infect., 6:1410-1417.

Leu J.H., Chang C.C., Wu J.L., Hsu C.W., Hirono I., Aoki T., Juan H.F., Lo C.F., Kou G.H. and H.C. Huang, 2007. Comparative analysis of differentially expressed genes in normal and white spot syndrome virus infected Penaeus monodon. BMC Genomics, 8:120.

Liu H., Soderhall K. and P. Jiravanichpaisal, 2009. Antiviral immunity in crustaceans. Fish Shellfish Immunol., 27:79-88.

Marañon G., Muñoz-Escassi B. Manley W., Garcia C., Cayado P., Sanchez de la Muela M, Olabarri B., Leon R. and E. Varra, 2008. The effect of methyl sulfonyl methane supplementation on markers of oxidative stress in sport horses following jumping exercise, Act. Vet. Scand., 50:45.

Mohankumar K. and P. Ramasamy, 2006. White spot syndrome virus infection decreases the activity of antioxidant enzyme in Fenneropenaeus indicus. Virus Res., 115:69-75.

Moret Y. and P. Schmid-Hempel, 2000. Survival for immunity: the price of immune system activation for bumblebee workers. Science, 290:1166-1168.

Namikoshi A., Wu J.L., Yamashita T., Nizhizawa T., Nishioka T., Arimoto M. and K. Muroga, 2004. Vaccination trials with Penaeus japonicus to induce resistance to white spot syndrome virus. Aquaculture, 229:25-35. 
Ortuño J., Esteban M.A. and J. Meseguer, 2000. High dietary intake of alpha-tocopherol enhances the non-specific immune response of gilthead seabream (Sparus aurata L). Fish Shellfish Immunol. 10: 293-307.

Robalino J., Bartlett T.C., Chapman R.W., Gross P.S., Browdy C.L. and G.W. Warr, 2007. Double-stranded RNA and antiviral immunity in marine shrimp: inducible host mechanisms and evidence for the evolution of viral counter-responses. Dev. Comp. Immunol., 31:539-547.

Schulenberg H., Boenisch C. and N. Michiels, 2007. How do invertebrates generate a highly specific innate immune response. Mol. Immunol., 44:33383344.

Teunissen O.S.P., Faber R., Booms G.H.R., Latscha T. and J.H. Boon, 1998. Influence of vaccination on vibriosis resistance of the giant black tiger shrimp Penaeus monodon (Fabricius). Aquaculture, 164:359-366.

Venegas C.A., Nonaka L., Mushiake K., Nizhizawa T. and K. Muroga, 2000. Quasi-immune response of Penaeus japonicus to penaeid rod-shaped DNA virus (PRDV). Dis. Aquat. Org., 42:83-89.

Watson L.F., Puttmann-Holgado R., Thomas F., Lamar D.L., Hughes M., Kondo M., Rebel V.I. and D. Schmucker, 2005. Extensive diversity of Igsuperfamily proteins in the immune system of insects. Science, 309:18741878.

Witteveldt J., Vlak J.M. and M.C.W. van Hulten, 2004. Protection of Penaeus monodon against white spot syndrome virus by oral vaccination. $J$. Virol., 78:2057-2061.

Zhang, S.M. and E.S. Loker, 2003. The FREP gene family in the snail Biomphalaria glabrata: additional members and evidence consistent with alternative splicing and FREP retrosequences. Dev. Comp. Immunol., 27:175187. 